International Journal of Engineering \& Technology, $7(3.2)(2018) 401-407$
International Journal of Engineering \& Technology
Website: www.sciencepubco.com/index.php/IJET
Research paper

\title{
Calculation of The Stability of the Form of Equilibrium of Discrete Systems
}

\author{
Oleksandr Shkurupiy ${ }^{1}$, Pavlo Mytrofanov², Vladislav Masiuk $^{3}$ \\ ${ }^{1}$ Poltava National Technical Yuri Kondratyuk University, Ukraine \\ ${ }^{2}$ Poltava National Technical Yuri Kondratyuk University, Ukraine \\ ${ }^{3}$ National University of Water and Environmental Engineering, Ukraine \\ *Corresponding author E-mail: shbm@ukr.net
}

\begin{abstract}
The paper presents an algorithm for calculating the stability of the equilibrium form of the first kind of compressed discrete systems by the displacements method in combination with the methods of iterations and bisection. The use of the methods makes it possible to effectively determine the minimum critical stress or strain at the first bifurcation and their corresponding form of stability loss, both for statically determined and statically undetermined systems. This approach, using matrix forms, makes it possible to significantly simplify the calculations of the analytical condition for the stability loss of compressed discrete systems (the stability loss equation), which has high orders, as well as to construct the form of stability loss corresponding to a critical load, that is, to solve the problem of loss of equilibrium stability. The calculation actually leads to solving a nonlinear transcendental equation, which is the equation of stability loss. The difficulty lies in the absence of an analytical solution of such an equation due to the presence of complex of Zhukovsky functions, which have transcendental functions in their structure. Such solution can be performed only with the use of numerical methods. This algorithm for calculating the loss of equilibrium of the first kind of compressed discrete systems by displacement in combination with the methods of iteration and bisection is implemented in the software complex "Persist" for PC in Windows OS. The program was approbated and implemented in the educational process at the Department of Structural and Theoretical Mechanics of Poltava National Technical Yuri Kondratyuk University during the training of specialists in engineering specialties.
\end{abstract}

Keywords: stability, critical state, critical load, critical stress, stability loss of the equilibrium form (deformation), bifurcation, form of stability loss, iteration, bisection.

\section{Introduction}

There are various methods for calculating the stability of the shape of the equilibrium of discrete systems, due to the large volume of computations associated with the solution of the analytical condition for the loss of equilibrium stability. The solution of the analytical condition for the stability loss of the equilibrium of compressed discrete systems, which has high orders, and the definition of the critical load of the form of stability loss, is one of the topical problems. The solution of the problem of calculating the analytical condition for stability loss of the equilibrium of compressed discrete systems, which have high orders, and the determination of the corresponding critical load of the form of stability loss, generated a large number of methods by many mathematicians (Krylov, Laverier, Danilevsky, Jacobi (iterations) [1 - 10] etc.). The calculation actually leads to solving a nonlinear transcendental equation, which is the equation of stability loss. The difficulty lies in the absence of analytical solution of such equation due to the presence of complex functions of Zhukovsky, which have transcendental functions in their structure. Such solution can be performed only with the use of numerical methods. This problem of calculating the analytical condition for stability loss of the equilibrium of compressed discrete systems, as well as the determination of the critical load of the form of stability loss, is proposed to solve by displacement, iteration and bisection methods, which makes it possible to significantly simplify calculations. The purpose of the work is to develop an algorithm and software for PC in Windows OS, which will enable students and engineers to automate calculations of stability of equilibrium forms of compressed discrete systems.

\section{Main Body}

Here is an algorithm for calculating the stability loss of the equilibrium form of the first kind of compressed discrete systems on the basis of the displacement method in expanded form in combination with the methods of inertia, bisection, chord in conjunction with refinement on iterations by methods of tangent [11].

1. Determine $t_{i}$ values for each $j$-th compressed rod.

$t_{j}^{2}=\frac{N_{j} \cdot l_{j}}{i_{j}}=\frac{N_{j} \cdot l_{j}^{2}}{E I_{j}}, \quad(j=1,2,3, \ldots, m)$

where $t$ - number of compressed rods.

2 . Accepted as a baseline $\left(t_{0}\right)$ any of the parameters $t_{j}$ and express the relationship between the parameters $t$.

$K_{0}=\frac{t_{0}}{t_{0}}=1 ; \ldots ; K_{j}=\frac{t_{j}}{t_{0}} ; \ldots ; K_{n}=\frac{t_{n}}{t_{0}}$ 
3. All parameters $t_{j}$ are expressed through basic $t_{0}$.

$t_{j}=K_{j} \cdot t_{0}$

4. We set the initial values of the basic parameter $t_{0}=0$, and determine the values of parameters in other rods, expressed in terms of the base.

5. We substitute in equations of stability loss the corresponding values of $t_{j}$, expressed in $t_{0}$, and calculate its value in the first iteration.

6 . We set $\Delta t_{0}$ the step of change the base parameter $t_{0}$, that is

$t_{0}=t_{0}+\Delta t_{0}$

7. We determine the value of the equation of stability loss on the next iteration in accordance with step 6 .

Iterations are performed until there is a change in the sign of the equation of stability loss $(D)$, that is, as it is depicted in the graph (Fig.1).

Execution of steps 5, 6,7 allows separating the value of the critical parameter with the error to the value of step $\Delta t_{0}$.

Using the iteration method, we determine the subinterval in which the first real root will be of the transcendental equation of stability loss of equilibrium forms.

The iterative process of solving a complex nonlinear equation of loss of equilibrium stability can also be implemented using the chord method in conjunction with the refinement on iterations by methods of tangential, bisection, and others. In this case, the search for the interval of existence of the first real root is carried out by the method of selection as for the method of dichotomy. The process of iterative refinement of the root continues until the root value reaches the specified accuracy.

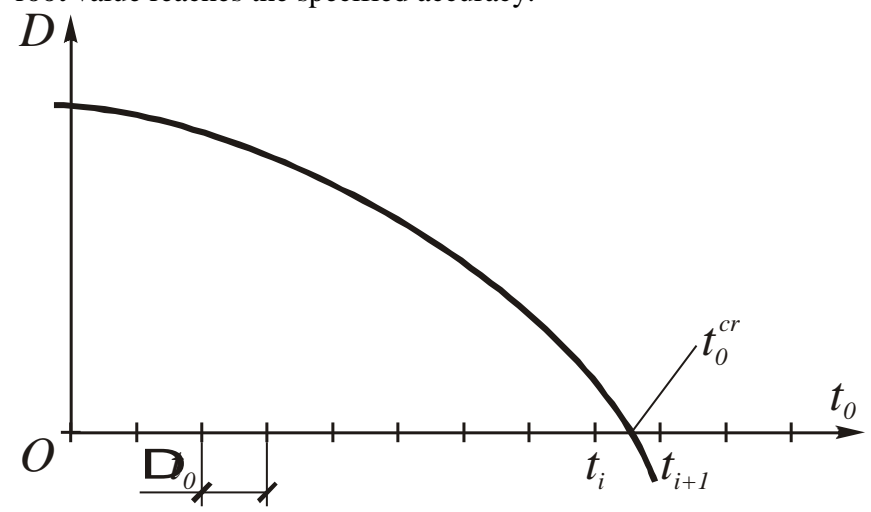

Fig. 1: Graph of the solution to the equation of stability loss

8. In the interval $t_{i}<t_{0}<t_{i+1}$ specify the value of the critical parameter $t_{0, c r}$ by the methods of bisection, tangent, and so on. The calculations are carried out as long as

$\left|t_{0, i}-t_{0, i+1}\right| \leq \varepsilon$

where $\varepsilon$ - the pre-specified accuracy of calculation of basic critical parameter $t_{0, c r}$.

9. It should be kept in mind that when selecting step $\Delta t_{0}$, you can skip the first critical root (the first least critical force). Therefore, when choosing it is necessary to accept as little as possible its initial value, ie $\Delta t_{0} \leq 0,5$.

To solve this engineering problem, the Persist software was developed and implemented on PC on the Department of Structural and Theoretical Mechanics of Poltava National Technical Yuri Kondratyuk University. This software is implemented in a modern compiler, contains several subroutines, utilities, which are combined and presented in the form of the same software package.

\section{General algorithm for calculating stability by the software "Persist".}

The calculation of the compressed frame on the stability of the equilibrium form involves forming a loss-stability equation independently.

Only after completing and checking the equation of stability loss as a nontrivial solution of a homogeneous system of canonical equations, a user uses a PC to solve stability of the equilibrium form according to the "Persist" software.

The calculation of the compressed discrete system on the stability of the equilibrium form actually reduces to the solution of the difficultly described nonlinear transcendental equation, which is the equation of stability loss. The difficulty lies in the absence of an analytical solution of such equation due to the presence of complex of Zhukovsky functions, which have transcendental functions in their structure. The arguments of the Zhukovsky functions are the values of the parameters of the compressed rods parameter

Generalized structural scheme of solving equation of stability loss:

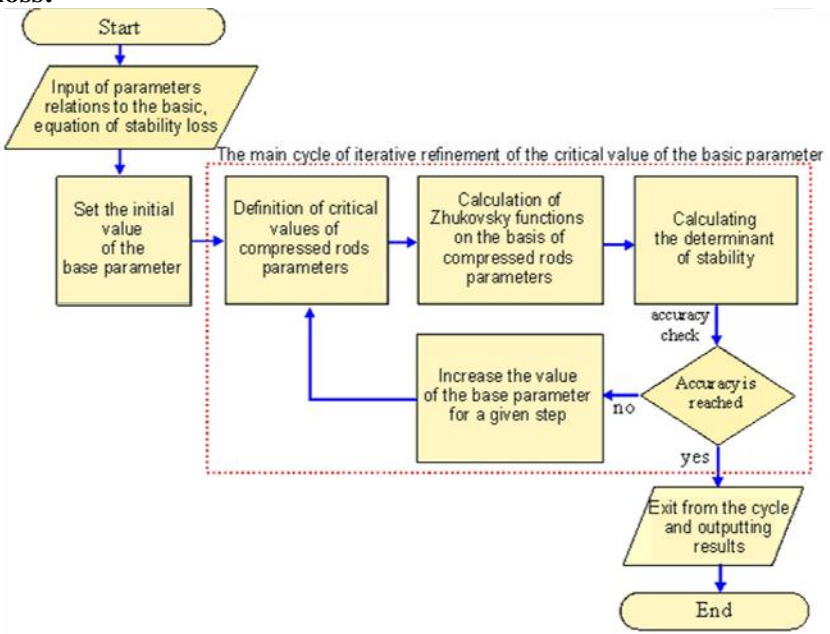

Fig. 2: Structural diagram of the algorithm for solving the equation of stability loss

The main points of describing the source data in the program «Persist».

1. Description of the compressed rods parameters.

The dialog for describing the parameters of the compressed rods is activated by pressing the corresponding button, Fig.3. In this case, it should be noted that only non-zero parameters are described, that is, the parameters of only compressed rods, rods with zero value of longitudinal force are not part of the ones described.

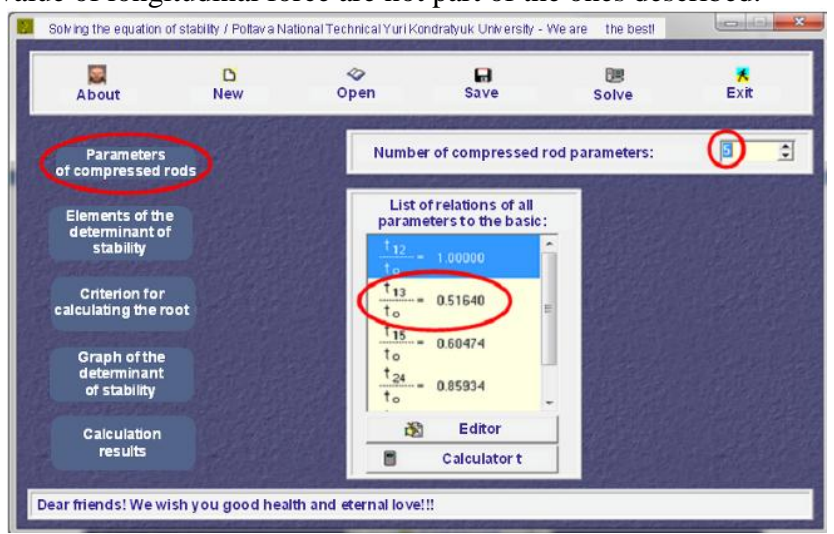

Fig. 3: Visual components for managing the description parameters of the compressed rods 
2. Introduction of elements of equation of stability loss of the equilibrium form.

Determination of the first real root of the equation of equilibrium of stability loss.

3. Criteria setting for iterative calculation.

As a precision criterion, the "Persist" program uses the so-called absolute criterion when the process of iterative refinement of the root stops when the absolute value of the desired root does not exceed the value of the given error, which is recommended to set in the range $10^{-6} \ldots 10^{-4}$. Fig.7 shows an activated menu item for adjusting the criteria for an iterative calculation.

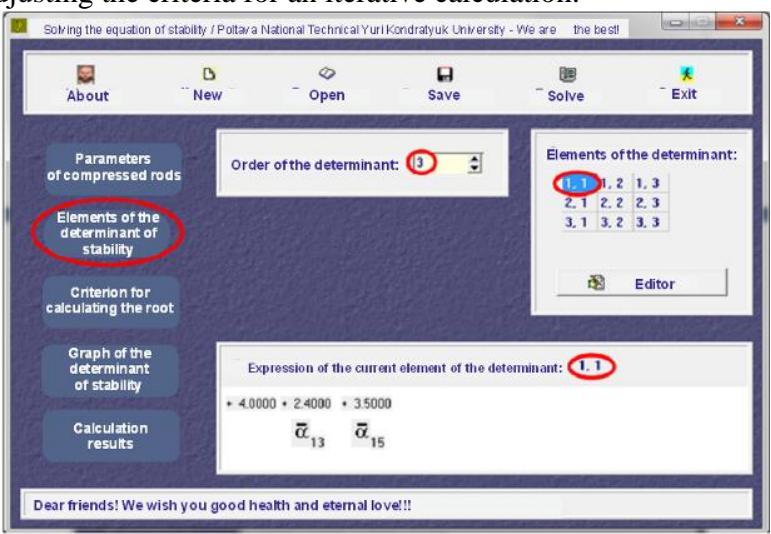

Fig. 4: Visual components of control description of equation elements of stability loss of the equilibrium form

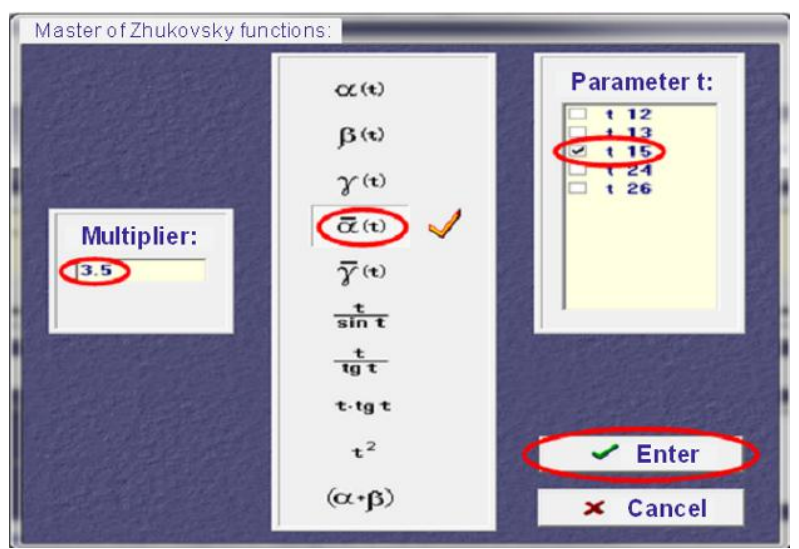

Fig. 5: Visual components of description control element 1.1 of equation of stability loss of the equilibrium form

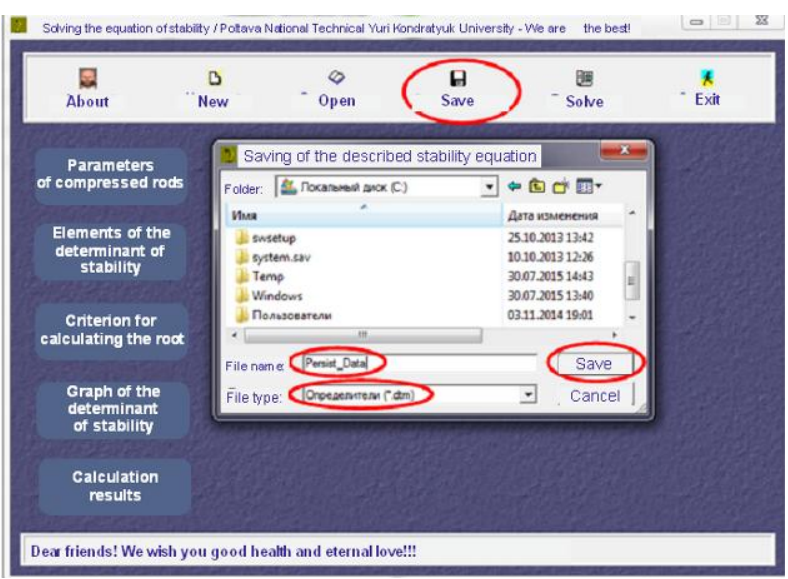

Fig. 6: Saving of the described equation of stability loss of the equilibrium form

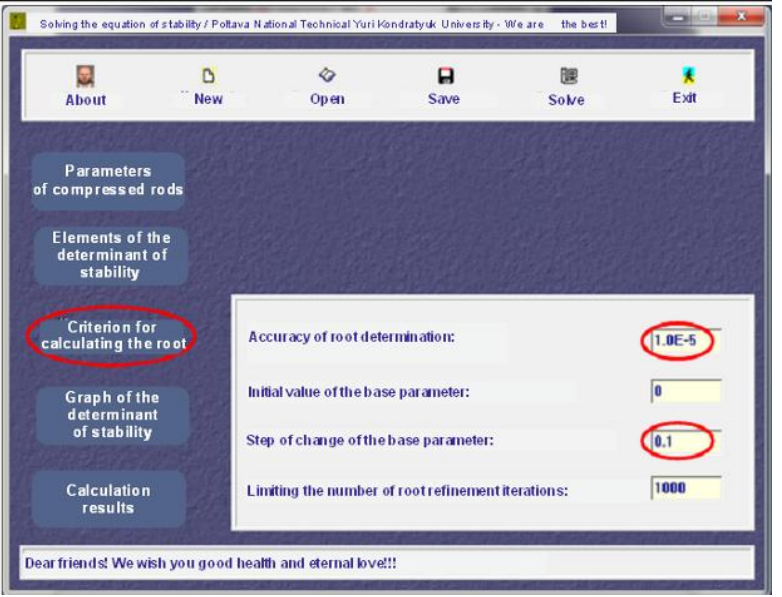

Fig. 7: Settings of criteria for iterative calculation

4. Directly solving the equation of stability in «Persist».

As noted above, solving the equation of stability loss of the equilibrium form involves the use of numerical methods. Thus, an iterative process of solving a complex nonlinear equation using the chord method in conjunction with refinement on its iterations by the method of bisection is organized in "Persist". The search for the interval of existence of the first root is carried out by the method of selection. The process of iterative refinement of the root continues until the root value reaches the given accuracy.

For a solution already described in the program "Persist" equation of stability loss of the equilibrium form, the user must use the item in the main menu "Solve", Fig.3.

5. Analysis and verification of calculation results.

As is known, transcendental equations can have not one root due to the presence of periodic functions. According to the physical content of the equation of stability loss, we are interested in the first root, which corresponds to the minimum critical value of the basic parameter of the compressed $\operatorname{rod} t_{0}^{\text {cr }}$. On its basis, the critical values of the parameters of all compressed rods are determined as: $t_{i}^{c r}=t_{0}^{c r} \cdot k_{i}$, where $k_{i}$ - is the coefficient of reduction of compressed rods lengths.

When numerically iterative solving of such an equation, it is necessary to focus attention on preventing the passage of the first root of the equation. To do this, one needs to analyze the results. As a rule, it is enough to visually assess the type of graph of the dependence of the determinant of stability of the equilibrium form from the value of the basic parameter $t_{0 c r}$ (Fig.8).

Fig. 9 and Fig.10 show the results of the solution of the equation of stability loss of the equilibrium form in table and graph forms.

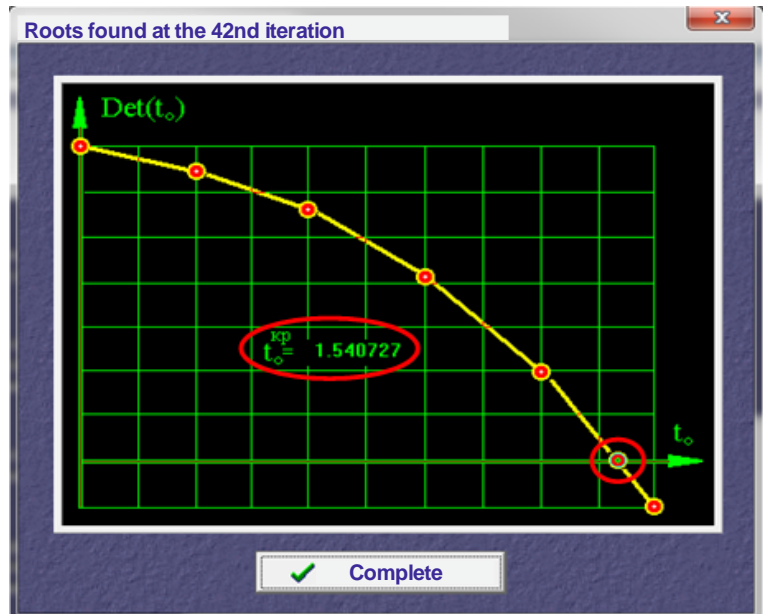

Fig. 8: Chart of the dependence of the determinant of resistance on the value of the base parameter 


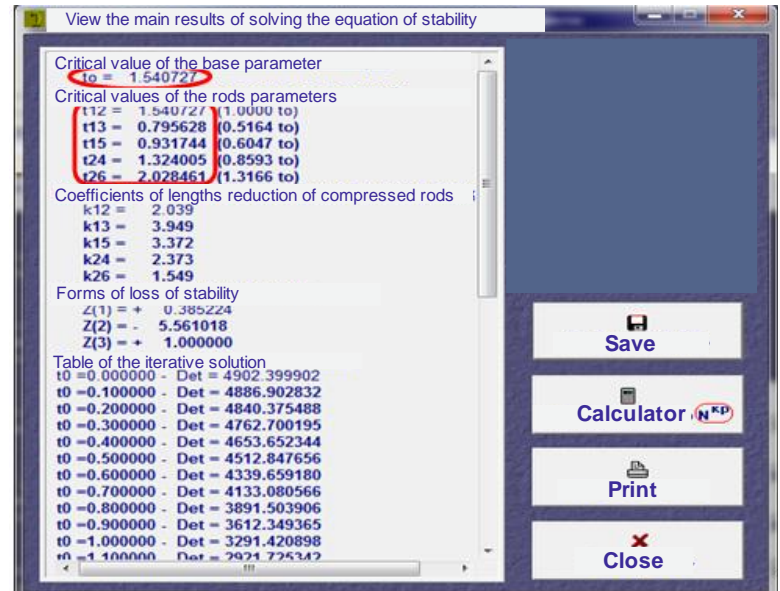

Fig. 9: View the results of the solution the equation of stability loss of the equilibrium form

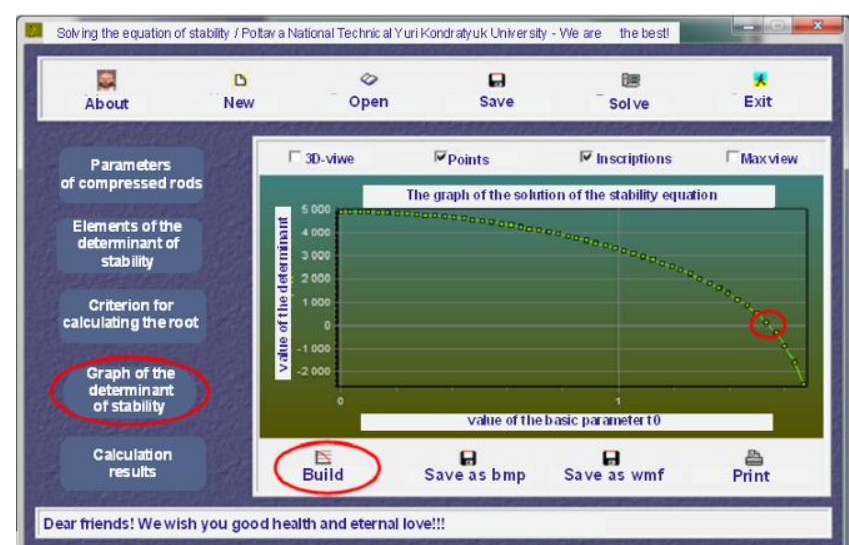

Fig. 10: Graphic representation of the solution of the equation of stability loss

The user is able to adjust the accuracy of the graphical display of the solution of the equation of stability loss (Fig.11). A calculator is available for the user which allows to calculate the values of critical longitudinal forces (Fig.12).

\begin{tabular}{l} 
Graph in a new range: \\
The initial value of the argument $(\mathrm{t} 0):$ \\
The final value of the argument $(\mathrm{t} 0):$ \\
Number of points \\
\hline Calculate \\
\hline
\end{tabular}

Fig. 11: Settings of the graphical representation of the solution of the equation of stability loss of the equilibrium form

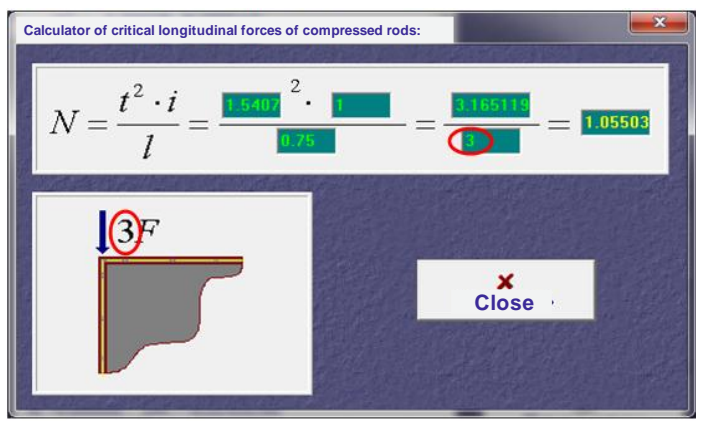

Fig. 12: Calculator for calculating critical longitudinal forces

6. Saving the results and getting a copy of them.

For the possibility of further analysis and processing of the results of calculations, "Persist" provides the possibility of saving them in a text file. To do this, one should use the appropriate menu item
"Save" in the viewport of the main results, see Fig.3. In this case, all actions of saving file are absolutely similar to those used in any Windows program (Fig.13).

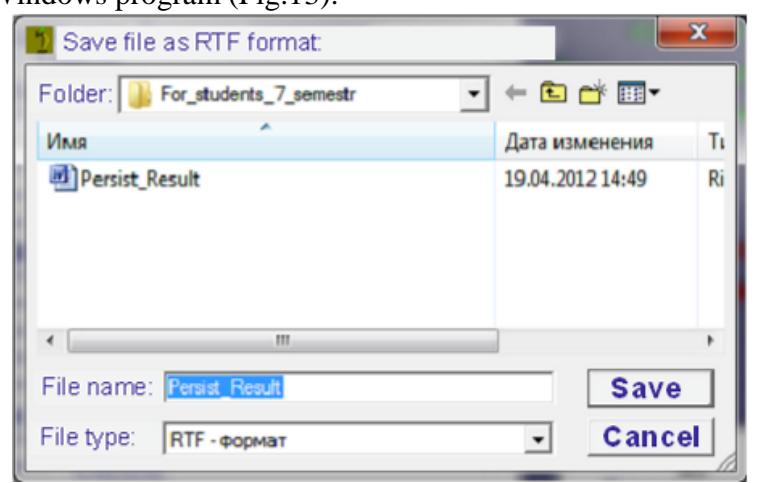

Fig. 13: Saving the results of solving the equation of stability loss in a tex file

A text file with results can be downloaded to any text editor to prepare for printing in a more user-friendly format.

7. Main properties of "Persist" software

Requirements for PC system resources. The program uses the Windows operating system. For ease of use, we recommend using monitors with a resolution of $800 \times 600$ pixels.

Software package Persist consist of files:

- Persist.exe (mandatory) - main program file;

- Persist.hlp (recommended) -help file for using "Persist";

- Persist.ini (optional) - initializing the values of the search criterion for the root equation of stability, can be created automatically;

- Persist.txt (optional) - file with a short description of the program;

- Demo*.dtm (optional) - demo files of the described equations of stability, which can be downloaded for calculation and analysis. Copyrights. The program can be distributed freely, and authors are not liable for any losses incurred as a result of using this program (data loss, financial loss, etc.).

Due to the constant improvement of the program, authors recommend that you follow the release number in the About menu. Installing software package «Persist» on PC. For correct operation of the program "Persist" you should install it on the hard disk of the PC. It is not desirable to launch the program directly from the $\mathrm{CD}$. This is due to the need in certain cases saving data on hard disk. To install the "Persist" program on a PC, you need to copy the "Persist" subdirectory to a PC hard disk.

Consider an example of calculating the stability of the equilibrium form of a compressed discrete system by the displacements method in expanded form. The relationship between the geometric dimensions of the rods and the stiffness on the bend:

$\frac{l}{h}=\frac{3}{4} ; \quad \frac{i_{1}}{i_{0}}=1 ; \quad \frac{i_{2}}{i_{0}}=1,3 ; \quad \frac{i_{3}}{i_{0}}=2,4 ; \quad \frac{i_{4}}{i_{0}}=3,5 ;$
$i_{1}=i_{0} ; \quad i_{2}=1,3 i_{0} ; \quad i_{3}=2,4 i_{0} ; \quad i_{4}=3,5 i_{0}$. 


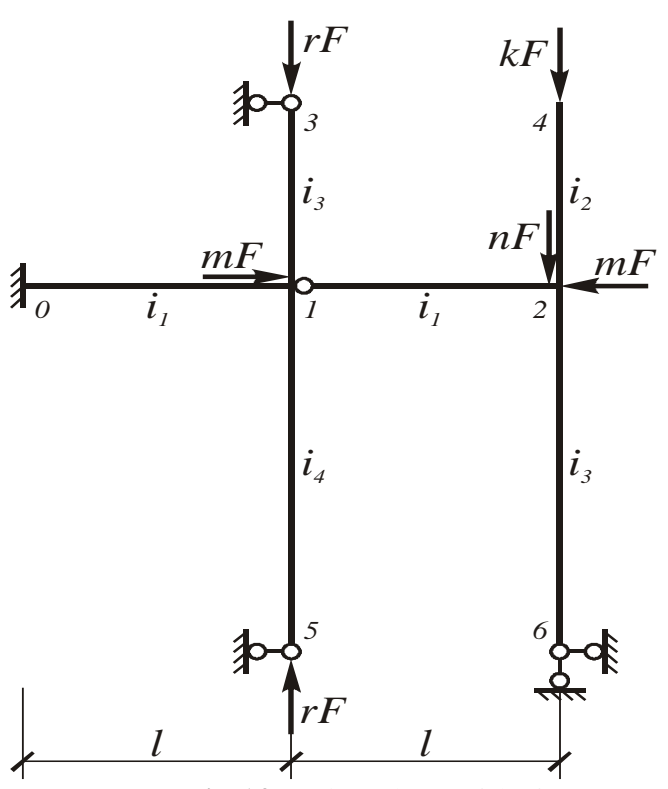

Fig. 14: Design scheme of the frame

1. Determination of the degree of kinematic uncertainty of the system

$n=n_{v}+n_{l}=2+1=3$

2. Determination of longitudinal forces in compressed frame rods

$N_{01}=0 ; \quad N_{12}=5 F ; \quad N_{31}=N_{15}=4 F ;$

$N_{42}=6 F ; \quad N_{26}=6 F+7 F=13 F$.

3. The basic system of the method of displacement
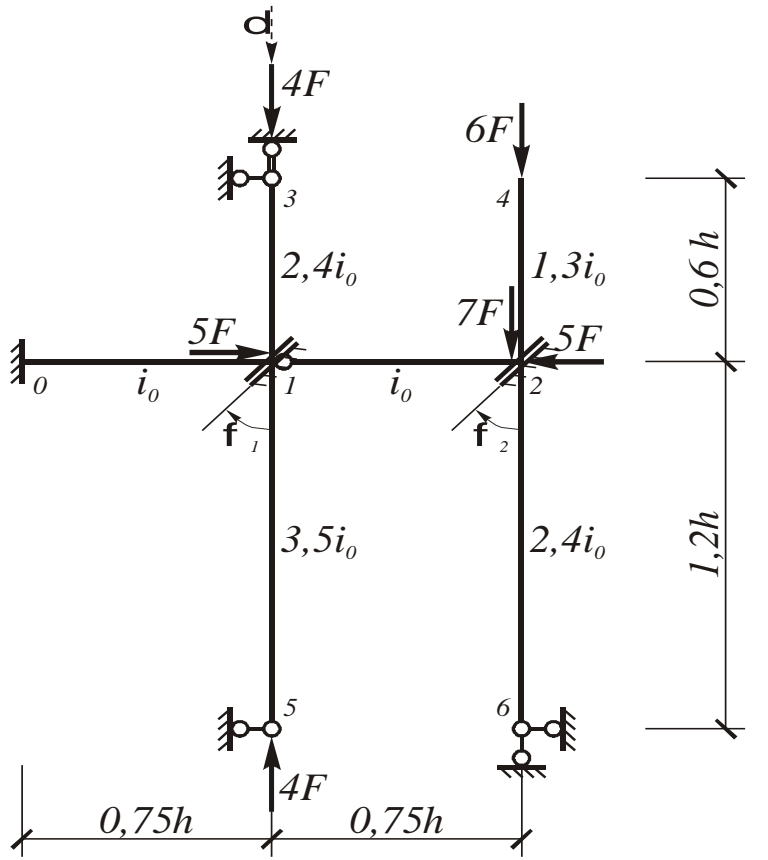

Fig. 15: The basic system of the method of displacement Basic unknowns: $\varphi_{1}, \varphi_{2}$ i $\delta$

4. Basic and additional equations of the method of displacement (expanded form)
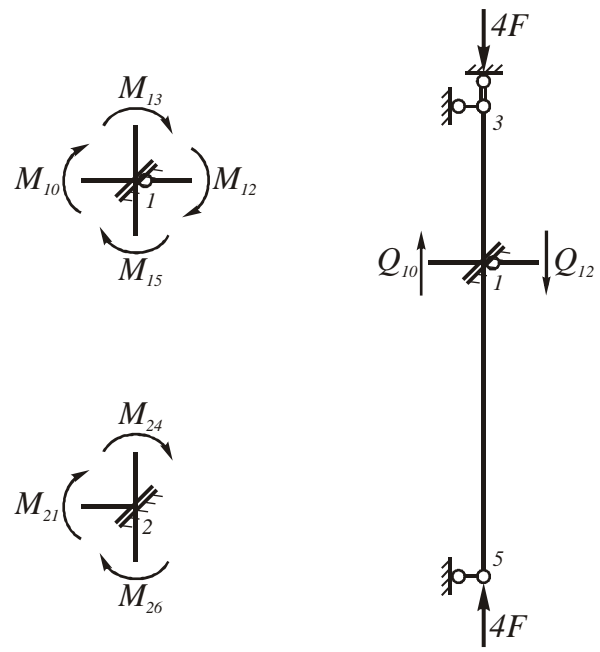

Basic equations:

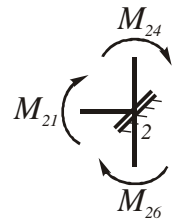

$\sum M_{1}=0 ; \quad M_{10}+M_{13}+M_{12}+M_{15}=0$

$\sum M_{2}=0 ; \quad M_{21}+M_{24}+M_{26}=0$

$\sum Y=0 ; \quad Q_{10}-Q_{12}=0$

$\left\{\begin{array}{l}M_{10}+M_{13}+M_{12}+M_{15}=0 \\ M_{21}+M_{24}+M_{26}=0 \\ Q_{10}-Q_{12}=0\end{array}\right.$

$=2 \cdot i_{0} \cdot\left[\alpha_{10} \cdot \varphi_{1}-(\alpha+\beta)_{10} \cdot \delta /(0,75 h)\right]$

Since $N_{01}=0$, then $\alpha_{10}=2$, and $\beta_{10}=1$.

Consequently $M_{10}=2 \cdot i_{0} \cdot\left[2 \cdot \varphi_{1}-4 \cdot \delta / h\right]$

$M_{13}=i_{13} \cdot \bar{\alpha}_{13} \cdot\left[\varphi_{1}-\psi_{13}\right]=2,4 \cdot i_{0} \cdot \bar{\alpha}_{13} \cdot \varphi_{1}$, and $M_{12}=0$

$M_{15}=i_{15} \cdot \bar{\alpha}_{15} \cdot\left[\varphi_{1}-\psi_{15}\right]=3,5 \cdot i_{0} \cdot \bar{\alpha}_{15} \cdot \varphi_{1}$

$\left(4+2,4 \cdot \bar{\alpha}_{13}+3,5 \cdot \bar{\alpha}_{15}\right) \cdot \varphi_{1} \cdot i_{0}+(-8) \cdot \delta / h \cdot i_{0}=0$

We express the bending moments of the second equation through the basic unknowns:

$M_{21}=i_{21} \cdot \bar{\alpha}_{21} \cdot\left[\varphi_{2}-\psi_{21}\right]=i_{0} \cdot \bar{\alpha}_{21} \cdot\left[\varphi_{2}+\delta /(0,75 h)\right]$

$M_{24}=-i_{24} \cdot t_{24} \cdot \operatorname{tg}_{24} \cdot \varphi_{2}=-1,3 \cdot i_{0} \cdot t_{24} \cdot \operatorname{tg} t_{24} \cdot \varphi_{2}$

$M_{26}=i_{26} \cdot \bar{\alpha}_{26} \cdot\left[\varphi_{2}-\psi_{26}\right]=2,4 \cdot i_{0} \cdot \bar{\alpha}_{26} \cdot \varphi_{2}$

$\left(\bar{\alpha}_{21}-1,3 \cdot t_{24} \cdot \operatorname{tgt}_{24}+2,4 \cdot \bar{\alpha}_{26}\right) \cdot \varphi_{2} \cdot i_{0}+\left(1,333 \cdot \bar{\alpha}_{21}\right) \cdot \delta / h \cdot i_{0}=0$

We express the transverse forces of the third equation through the main unknowns: 
$Q_{10}=-2 \cdot i_{10} / l \cdot\left[(\alpha+\beta)_{10} \cdot\left(\varphi_{1}+\varphi_{0}\right)-\gamma_{10} \cdot \psi_{10}\right]=$

$=-2 \cdot i_{0} /(0,75 h) \cdot\left[3 \cdot \varphi_{1}-6 \cdot \delta /(0,75 h)\right]$

$Q_{12}=-i_{12} / l \cdot\left[\bar{\alpha}_{12} \cdot \varphi_{2}-\bar{\gamma}_{12} \cdot \delta / l\right]=$

$=-i_{0} /(0,75 h) \cdot\left[\bar{\alpha}_{12} \cdot \varphi_{2}+\bar{\gamma}_{12} \cdot \delta /(0,75 h)\right]$

$(-8) \cdot \varphi_{1} / h \cdot i_{0}+\left(1,333 \cdot \bar{\alpha}_{21}\right) \cdot \varphi_{2} / h \cdot i_{0}+$

$+\left(21,333+1,778 \cdot \bar{\gamma}_{21}\right) \cdot \delta / h^{2} \cdot i_{0}=0$

The system of equations of the method of displacement in expanded form:

$$
\left\{\begin{array}{l}
\left(4+2,4 \cdot \bar{\alpha}_{13}+3,5 \cdot \bar{\alpha}_{15}\right) \cdot \varphi_{1}+0 \cdot \varphi_{2}-8 \cdot \delta / h=0 ; \\
0 \cdot \varphi_{1}+\left(\bar{\alpha}_{21}-1,3 \cdot t_{24} \cdot \operatorname{tgt} t_{24}+2,4 \cdot \bar{\alpha}_{26}\right) \cdot \varphi_{2}+\left(1,333 \cdot \bar{\alpha}_{21}\right) \cdot \delta / h=0 \\
-8 \cdot \varphi_{1}+\left(1,333 \cdot \bar{\alpha}_{21}\right) \cdot \varphi_{2}+\left(21,333+1,778 \cdot \bar{\gamma}_{21}\right) \cdot \delta / h=0 .
\end{array}\right.
$$

Determination of the relationship between the parameters $t$ of the compressed rods:

$$
\begin{aligned}
& t_{i}=t_{a b}=\sqrt{\frac{N_{a b} \cdot l_{a b}}{i_{a b}}} ; \quad t_{0}=t_{12}=\sqrt{\frac{5 F}{i_{0}} \cdot 0,75 h} ; \\
& t_{1}=t_{13}=\sqrt{\frac{4 F}{2,4 i_{0}} \cdot 0,6 h} ; \quad t_{2}=t_{15}=\sqrt{\frac{4 F}{3,5 i_{0}} \cdot 1,2 h} ; \\
& t_{3}=t_{24}=\sqrt{\frac{6 F}{1,3 i_{0}} \cdot 0,6 h} ; \quad t_{4}=t_{26}=\sqrt{\frac{13 F}{2,4 i_{0}} \cdot 1,2 h} ; \\
& K_{i}=\frac{t_{i}}{t_{0}} ; \quad K_{0}=1 ; \quad K_{1}=\sqrt{\frac{4 F \cdot 0,6 h \cdot i_{0}}{2,4 i_{0} \cdot 5 F \cdot 0,75 h}} \cong 0,516398 ; \\
& K_{2}=\sqrt{\frac{4 F \cdot 1,2 h \cdot i_{0}}{3,5 i_{0} \cdot 5 F \cdot 0,75 h}} \cong 0,604743 \text {; } \\
& K_{3}=\sqrt{\frac{6 F \cdot 0,6 h \cdot i_{0}}{1,3 i_{0} \cdot 5 F \cdot 0,75 h}} \cong 0,859338 \text {; } \\
& K_{4}=\sqrt{\frac{13 F \cdot 1,2 h \cdot i_{0}}{2,4 i_{0} \cdot 5 F \cdot 0,75 h}} \cong 1,316561 \text {. }
\end{aligned}
$$

5. Output data for calculating the equation of stability loss on a PC on the given algorithm.

The relationship between the parameters t:

$t_{0}=t_{12} ; \quad t_{1}=t_{13} ; \quad t_{2}=t_{15} ; \quad t_{3}=t_{24} ; \quad t_{4}=t_{26} ;$

$K_{0}=1 ; \quad K_{1}=0,516398 ; \quad K_{2}=0,604743$;

$K_{3}=0,859338 ; \quad K_{4}=1,316561$.

Equation the loss of equilibrium stability (upper determinant triangle):

$$
\left|\begin{array}{ccc}
4+2,4 \cdot \bar{\alpha}_{13}+3,5 \cdot \bar{\alpha}_{15} & 0 & -8 \\
& \bar{\alpha}_{21}-1,3 \cdot t_{24} \cdot \operatorname{tgt}_{24}+2,4 \cdot \bar{\alpha}_{26} & 1,333 \cdot \bar{\alpha}_{21} \\
& & 21,333+1,778 \cdot \bar{\gamma}_{21}
\end{array}\right|=0
$$

\begin{tabular}{|c|c|}
\hline $\mathrm{t}_{0}$ & Det \\
\hline 0.000000 & 4902.399902 \\
\hline 0.100000 & 4886.902832 \\
\hline 0.200000 & 4840.375488 \\
\hline 0.300000 & 4762.700195 \\
\hline 0.400000 & 4653.652344 \\
\hline 0.500000 & 4512.847656 \\
\hline 0.600000 & 4339.659180 \\
\hline 0.700000 & 4133.080566 \\
\hline 0.800000 & 3891.503906 \\
\hline 0.900000 & 3612.349365 \\
\hline 1.000000 & 3291.420898 \\
\hline 1.100000 & 2921.725342 \\
\hline 1.200000 & 2491.159424 \\
\hline 1.300000 & 1977.557617 \\
\hline 1.400000 & 1336.789063 \\
\hline 1.500000 & 469.061646 \\
\hline 1.550000 & -121.025391 \\
\hline 1.525000 & 192.218048 \\
\hline 1.537500 & 40.719765 \\
\hline 1.543750 & -38.789200 \\
\hline 1.540625 & 1.295279 \\
\hline 1.542187 & -18.663136 \\
\hline 1.541406 & -8.663139 \\
\hline 1.541016 & -3.678754 \\
\hline 1.540820 & -1.190446 \\
\hline 1.540723 & 0.052739 \\
\hline 1.540771 & -0.568773 \\
\hline 1.540747 & -0.257997 \\
\hline 1.540735 & -0.102624 \\
\hline 1.540729 & -0.024941 \\
\hline 1.540726 & 0.013899 \\
\hline 1.540727 & -0.005521 \\
\hline 1.540726 & 0.004189 \\
\hline 1.540727 & -0.000666 \\
\hline
\end{tabular}

Results of calculation:

The values of the critical parameters $t$ of the compressed rods are:

$t_{01}=2,503462 ; \quad t_{12}=1,269506 ; \quad t_{23}=1,526861 ;$

$t_{35}=4,231602 ; \quad t_{46}=2,448636$.

Forms of stability loss:

$$
\begin{aligned}
& Z(1)=\phi_{1}=0,385224 \\
& Z(2)=\phi_{2}=-5,561034 \\
& Z(3)=\delta / h=1,000000
\end{aligned}
$$

able 1. Iterative solution the equation of stability loss of the equilibrium form

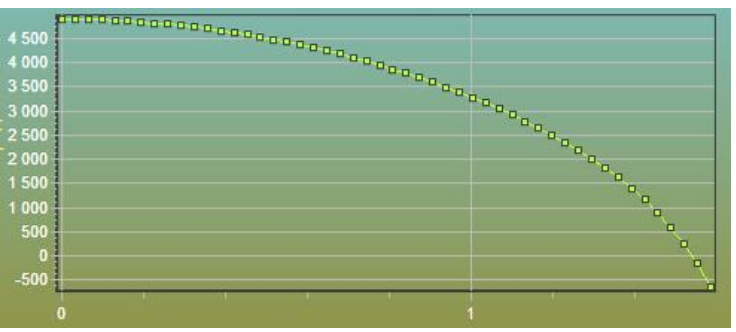

Fig. 16: Graph of the solution the equation of stability loss

6. Determination of longitudinal critical forces in compressed rods

$N_{a b}^{c r}=\frac{\left(t_{a b}^{c r}\right)^{2} \cdot i_{a b}}{l_{a b}}$.

$N_{12}^{c r}=\frac{\left(t_{12}^{c r}\right)^{2} \cdot i_{0}}{0,75 h}=\frac{1,540727^{2} \cdot i_{0}}{0,75 h}=3,165 \frac{i_{0}}{h}[\kappa N]$

$N_{13}^{c r}=\frac{\left(t_{13}^{c r}\right)^{2} \cdot 2,4 i_{0}}{0,6 h}=\frac{0,795628^{2} \cdot 2,4 i_{0}}{0,6 h}=2,532 \frac{i_{0}}{h}[\kappa N]$

$N_{15}^{c r}=\frac{\left(t_{15}^{c r}\right)^{2} \cdot 3,5 i_{0}}{1,2 h}=\frac{0,931744^{2} \cdot 3,5 i_{0}}{1,2 h}=2,532 \frac{i_{0}}{h}[\kappa N]$

$N_{24}^{c r}=\frac{\left(t_{24}^{c r}\right)^{2} \cdot 1,3 i_{0}}{0,6 h}=\frac{1,324005^{2} \cdot 1,3 i_{0}}{0,6 h}=3,798 \frac{i_{0}}{h}[\kappa N]$ 
$N_{26}^{c r}=\frac{\left(t_{26}^{c r}\right)^{2} \cdot 2,4 i_{0}}{1,2 h}=\frac{2,028461^{2} \cdot 2,4 i_{0}}{1,2 h}=8,229 \frac{i_{0}}{h}[\kappa N]$

7. Determination of critical load

$$
\begin{aligned}
& F_{12}^{c r}=\frac{N_{12}^{c r}}{5}=0,633 \frac{i_{0}}{h} \quad[\kappa N] \\
& F_{13}^{c r}=\frac{N_{13}^{c r}}{4}=0,633 \frac{i_{0}}{h} \quad[\kappa N] \\
& F_{15}^{c r}=\frac{N_{15}^{c r}}{4}=0,633 \frac{i_{0}}{h} \quad[\kappa N] \\
& F_{24}^{c r}=\frac{N_{24}^{c r}}{6}=0,633 \frac{i_{0}}{h} \quad[\kappa N] \\
& F_{26}^{c r}=\frac{N_{26}^{c r}}{13}=0,633 \frac{i_{0}}{h} \quad[\kappa N] \\
& F_{\text {mid }}^{c r}=\frac{5 \cdot 0,633}{5} \cong 0,633 \frac{i_{0}}{h} \quad[\kappa N] \\
& \varepsilon=\left|\frac{0,633-0,633}{0,633}\right| \cdot 100 \%=0 \%
\end{aligned}
$$

8. Determination of the coefficients of reducing the lengths of the compressed rods

$$
\begin{aligned}
& \mu_{a b}=\frac{\pi}{t_{a b}^{c r}} \\
& \mu_{12}=\frac{\pi}{t_{12}^{c r}}=\frac{\pi}{1,540727}=2,039>0,7 \\
& \mu_{13}=\frac{\pi}{t_{13}^{c r}}=\frac{\pi}{0,795628}=3,949>0,7 \\
& \mu_{15}=\frac{\pi}{t_{15}^{c r}}=\frac{\pi}{0,931744}=3,372>0,7 \\
& \mu_{24}=\frac{\pi}{t_{24}^{c r}}=\frac{\pi}{1,324005}=2,373>2 \\
& \mu_{26}=\frac{\pi}{t_{26}^{c r}}=\frac{\pi}{2,028461}=1,549>0,7
\end{aligned}
$$

9. Estimated lengths of compressed rods

$$
\begin{aligned}
& l_{0}^{a b}=\mu_{a b} \cdot l_{a b} \\
& l_{0}^{12}=\mu_{12} \cdot 0,75 h=2,039 \cdot 0,75 h=1,529 h \quad[\mathrm{~m}] \\
& l_{0}^{13}=\mu_{13} \cdot 0,6 h=3,949 \cdot 0,6 h=2,369 h \quad[\mathrm{~m}] \\
& l_{0}^{15}=\mu_{15} \cdot 1,2 h=3,372 \cdot 1,2 h=4,046 h \quad[\mathrm{~m}] \\
& l_{0}^{24}=\mu_{24} \cdot 0,6 h=2,373 \cdot 0,6 h=1,424 h \quad[\mathrm{~m}]
\end{aligned}
$$

$l_{0}^{26}=\mu_{26} \cdot 1,2 h=1,549 \cdot 1,2 h=1,859 h \quad[m]$

10. Forms of stability loss and construction of a deformed frame scheme

We accept $\delta / h=1$.

$\left\{\begin{array}{l}r_{11} \cdot \varphi_{1}+r_{12} \cdot \varphi_{2}=R_{1} \\ r_{21} \cdot \varphi_{1}+r_{22} \cdot \varphi_{2}=R_{2}\end{array}\right.$

$\left\{\begin{array}{l}\left(4+2,4 \cdot \bar{\alpha}_{13}+3,5 \cdot \bar{\alpha}_{15}\right) \cdot \varphi_{1}=8 ; \\ \left(\bar{\alpha}_{21}-1,3 \cdot t_{24} \cdot \operatorname{tgt} 24+2,4 \cdot \bar{\alpha}_{26}\right) \cdot \varphi_{2}=1,333 \cdot \bar{\alpha}_{21} .\end{array}\right.$

$Z(1)=\varphi_{1}=0,385224$

$Z(2)=\varphi_{2}=-5,561034$

$Z(3)=\delta / h=1,000000$.

\section{Conclusions}

The algorithm and program complex "Persist" for PC in Windows OS are developed. This computer program is implemented in a modern compiler, contains several subroutines (utilities), which are combined and presented in the form of the same software complex. It will enable students and engineers to automate calculations of the stability of the equilibrium form of compressed discrete systems, calculate critical loads (stresses) and determining the corresponding principal forms of oscillations. The program has been approbated and implemented in the educational process at the Poltava National Technical Yuri Kondratyuk University during the training of specialists in engineering specialties.

\section{References}

[1] Ray Hulse, Jack Cain, Structural Mechanics, Palgrave, London, (1991), 294 p., https://doi.org/10.1007/978-1-349-11897-7

[2] Keith D. Hjelmstad, Fundamentals of Structural Mechanics, Springer, Boston, MA, (2005), 480 p., https://doi.org/10.1007/b101129

[3] Friedel Hartmann, The Mathematical Foundation of Structural Mechanics, Springer, Berlin, Heidelberg, (1985), 371 p., https://doi.org/10.1007/978-3-642-82401-2

[4] Walter Lacarbonara, Nonlinear Structural Mechanics, Springer, Boston, MA, (2013), 802 p., https://doi.org/10.1007/978-1-4419-1276-3

[5] Smyrnov A.F., Aleksandrov A.V., Lashchenykov B.Ya., Shaposhnykov N.N. Stroitel'naya mekhanika. Dynamika i ustoichivost' sooruzheniy [Structural mechanics. Dynamics and stability of structures]. - Moscow: Stroyyzdat, 1984. - 415 p. [in Russian].

[6] Kyselev V.A. Stroitel'naya mekhanika. Spetsial'niy kurs [Structural mechanics. Special course]. - Moscow: Stroyyzdat, 1980. - 616 p. [in Russian].

[7] Bazhenov V.A. Perel'muter A.V., Shyshov O.V. Budivel'na mekhanika Kompyuterni tekhnolohiyi: pidruchnyk [Structural Mechanics. Computer Technology: Tutorial]. - Kyiv.: Karavela, 2009. - 696 p. [in Ukrainian].

[8] Bazhenov V.A. Dekhtyaryuk Ye.S. Budivel'na mekhanika. Dynamika sporud: navch. posibnyk [Structural Mechanics. Dynamics of structures: Tutorial]. - K.: IZMN, 1998. - 208 p. [in Ukrainian].

[9] Faddeev D.K., Faddeeva V.N. Vichyslytel'nie metody lyneynoy alhebry [Computational methods of linear algebra]. - Moscow: Hosudarstvennoe yzdatel'stvo fyzyko-matematycheskoy lyteratury, 1960. - $656 \mathrm{p}$. [in Russian].

[10] Smith P. An Introduction to Structural Mechanics / P. Smith. - Palgrave Macmillan, 2001. - $368 \mathrm{p}$.

[11] Shkurupiy O.A. Stiykist' formy rivnovahy ta dynamika dyskretnykh system: navchal'nyy posibnyk [Stability of equilibrium and dynamics of discrete systems: Tutorial]. - Poltava: PoltNTU, 2015. - 228 p. [in Ukrainian] 